\title{
Phase separation of EML4-ALK in firing downstream signaling and promoting lung tumorigenesis
}

\author{
Zhen Qin ${ }^{1,2}$, Honghua Sun ${ }^{1,2}$, Meiting Yue ${ }^{1,2}$, Xinwen Pan ${ }^{1,3}$, Liang Chen ${ }^{4}$, Xinhua Feng $\mathbb{1}^{5}$, Xiumin Yan ${ }^{1,2}$,
}

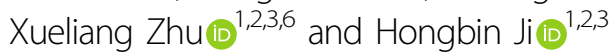

\begin{abstract}
EML4-ALK fusion, observed in about 3\%-7\% of human lung adenocarcinoma, is one of the most important oncogenic drivers in initiating lung tumorigenesis. However, it still remains largely unknown about how EML4-ALK fusion exactly fires downstream signaling and drives lung cancer formation. We here find that EML4-ALK variant 1 (exon 1-13 of EML4 fused to exon 20-29 of ALK) forms condensates via phase separation in the cytoplasm of various human cancer cell lines. Using two genetically engineered mouse models (GEMMs), we find that EML4-ALK variant 1 can drive lung tumorigenesis and these murine tumors, as well as primary tumor-derived organoids, clearly show the condensates of EML4-ALK protein, further supporting the findings from in vitro study. Mutation of multiple aromatic residues in EML4 region significantly impairs the phase separation of EML4-ALK and dampens the activation of the downstream signaling pathways, especially the STAT3 phosphorylation. Importantly, it also significantly decreases cancer malignant transformation and tumor formation. These data together highlight an important role of phase separation in orchestrating EML4-ALK signaling and promoting tumorigenesis, which might provide new clues for the development of clinical therapeutic strategies in treating lung cancer patients with the EML4-ALK fusion.
\end{abstract}

\section{Introduction}

Non-small cell lung cancer (NSCLC) is one of the most common cancers worldwide with high incidence and mortality ${ }^{1}$. According to the pathological classification, NSCLC can be categorized into three subtypes: adenocarcinoma (ADC), squamous cell carcinoma (SCC), and large cell carcinoma (LCC) ${ }^{2}$. NSCLC is frequently associated with oncogenic driver mutations which significantly contribute to tumorigenesis and cancer progression. For example, oncogenic mutations of epidermal growth factor receptor (EGFR), v-Ki-ras2 Kirsten

\footnotetext{
Correspondence: Xiumin Yan (yanx@sibcb.ac.cn) or Xueliang Zhu (xlzhu@sibcb. ac.cn) or Hongbin Ji (hbji@sibcb.ac.cn)

${ }^{1}$ State Key Laboratory of Cell Biology, Shanghai Institute of Biochemistry and Cell Biology, Center for Excellence in Molecular Cell Science, Chinese Academy of Sciences, Shanghai, China

'University of Chinese Academy of Sciences, Beijing, China

Full list of author information is available at the end of the article

These authors contributed equally: Zhen Qin, Honghua Sun
}

rat sarcoma viral oncogene (KRAS), and anaplastic lymphoma kinase (ALK) fusions are the most frequent oncogenic drivers in NSCLC ${ }^{3}$.

The EML4-ALK fusion was initially discovered in Japanese NSCLC patients by Soda and colleagues in $2007^{4}$. Later study showed that about $3 \%-7 \%$ of NSCLC patients harbor EML4-ALK fusion ${ }^{5}$. ALK belongs to the receptor tyrosine kinase (RTK) family and the full length of ALK protein contains 1620 amino acids ${ }^{6}$. The ALK protein is comprised of three domains, including an extracellular domain (ECD), a transmembrane domain, and an intracellular domain (ICD) ${ }^{7}$. ALK expression is physiologically limited to embryonic stage, exclusively in the embryonic nervous systems, small intestine, and testis $^{8}$. As a fusion partner of ALK, echinoderm microtubuleassociated protein-like 4 (EML4) belongs to the echinoderm microtubule-associated protein-like family. EML4 comprises an $\mathrm{N}$-terminal basic region, a hydrophobic

\section{(c) The Author(s) 2021}

(c) (i) Open Access This article is licensed under a Creative Commons Attribution 4.0 International License, which permits use, sharing, adaptation, distribution and reproduction cc) in any medium or format, as long as you give appropriate credit to the original author(s) and the source, provide a link to the Creative Commons license, and indicate if changes were made. The images or other third party material in this article are included in the article's Creative Commons license, unless indicated otherwise in a credit line to the material. If material is not included in the article's Creative Commons license and your intended use is not permitted by statutory regulation or exceeds the permitted use, you will need to obtain permission directly from the copyright holder. To view a copy of this license, visit http://creativecommons.org/licenses/by/4.0/. 
echinoderm microtubule-associated protein-like protein (HELP) domain, and a tryptophan-aspartic acid (WD) repeat domain 9 . Previous study showed that the EML4 protein might be involved in the process of microtubule formation'. There are at least 15 EML4-ALK variants have been reported thus far and they uniformly contain the entire intracellular kinase domain of ALK which is encoded by exons $20-29^{10}$. Among all the EML4-ALK variants, EML4-ALK variant 1 is the most frequent form that accounts for about $43 \%$ patients $^{11}$.

Unlike ALK as a membrane protein, the EML4-ALK fusion lacks the transmembrane domain and frequently localizes in the cytoplasm or microtubules ${ }^{10}$. By far two genetically engineered mouse models (GEMMs) of EML4-ALK have been established. Soda et al. generated the EML4-ALK mice, in which the EML4-ALK expression is driven by the surfactant protein C (SPC) promoter $^{12}$. In another study, Pyo et al. developed a transgenic mouse model with the tamoxifen-inducible EML4-ALK expression ${ }^{13}$. The major downstream signaling pathways of EML4-ALK fusion include the mitogen-activated protein kinase (MAPK), the phosphoinositide-3-kinase (PI3K), and the signal transducer and activator of transcription 3 (STAT3) pathways ${ }^{14}$. Moreover, Zhang et al. recently found that ALK could inhibit TGF- $\beta$ signaling through phosphorylating SMAD4 on tyrosine residue $95^{15}$. Activation of these signaling pathways could promote tumor cell survival, proliferation, and angiogenesis ${ }^{16}$.

Recent studies have highlighted the important role of protein phase separation in the formation of nonmembranous organelles or compartments in both the nucleus and cytoplasm ${ }^{17-20}$. Phase separation is not only a simple physicochemical process but also regulates biological functions and activities ${ }^{20,21}$. However, whether protein phase separation could impact tumorigenesis, remains unclear.

We here demonstrate that EML4-ALK variant 1 forms condensates via phase separation in human cancer cell lines, murine lung tumors as well as tumor-derived organoids. Our data show that the phase separation of EML4-ALK is important for firing downstream signalings, especially the STAT3 phosphorylation, and promoting tumorigenesis.

\section{Results}

Phase separation of EML4-ALK variant 1 in human cancer cell lines

To understand the localization and protein properties of EML4-ALK, we transiently expressed GFP-EML4-ALK variant 1 in HeLa cells, a commonly-used human cancer cell line. We clearly observed multiple near-spherical condensates positive for GFP-EML4-ALK in the cytoplasm (Fig. 1a). Intrigued by this finding, we next tested whether these condensates were formed through the liquid-liquid phase separation (LLPS). Through living cell imaging analyses, we found that the GFP-EML4-ALK condensates were able to undergo fusion, indicative of their liquid properties (Fig. 1b and Supplementary Movie S1). Fluorescence recovery after photobleaching (FRAP) assays revealed protein exchanges between the liquid droplets and the surroundings despite of a low exchange efficiency (Fig. 1c, d and Supplementary Fig. S1). As EML4-ALK is known as an important oncogenic driver in lung tumorigenesis, we overexpressed GFP-EML4-ALK variant 1 in BEAS-2B cells, a non-transformed human bronchial epithelial cell line and observed similar condensate formation (Fig. 1e). More importantly, when H2228 cells, an EML4-ALK fusion-containing lung cancer cell line, were immunostained with an anti-ALK antibody, we observed similar spherical condensates (Fig. 1f), suggesting that endogenous EML4-ALK also undergoes LLPS. We also performed the FRAP assays in BEAS-2B and H2228 cells and observed similar low protein exchange efficiency (Supplementary Fig. S2a, b). These data together suggest that EML4-ALK has an intrinsic capacity to phase separate into liquid-like condensates in various human cancer cell lines.

\section{Phase separation of EML4-ALK in murine tumors and organoids}

To test whether the phase separation of EML4-ALK fusion exists in vivo, we took advantage of two GEMMs. In the first mouse model, we delivered the lentivirus carrying EML4-ALK variant 1 together with Cre into the $\operatorname{Trp} 53^{\text {flox flox }}$ mice through nasal inhalation as previously described $^{22}$ (Fig. 2a). After 28 weeks of viral treatment $\left(1 \times 10^{6} \mathrm{PFU}\right)$, these mice were sacrificed for pathological analysis and 3D tumor organoids culture (Fig. 2a, c). These tumors displayed high expression of EML4-ALK (Fig. 2b). Importantly, the EML4-ALK condensates were clearly detectable in these murine lung tumors (Fig. 2d) and tumor-derived organoids (Fig. 2e). In the second mouse model, we integrated the loxp-stop-loxpEML4-ALK variant 1 transgene into the Rosa26 locus of C57BL/6 mice (Fig. 3a). After 5 weeks of nasal inhalation with $2 \times 10^{6}$ PFU Ad-Cre, the mice were sacrificed for further analyses (Fig. 3b, d). Consistently, we found strong EML4-ALK expression in tumor areas (Fig. 3c). Obvious condensate formation of EML4-ALK proteins was observed in both murine lung tumors (Fig. 3e) and tumorderived organoids (Fig. 3f). These data together provide in vivo evidence in supporting the phase separation of EML4-ALK.

\section{Phase separation of EML4-ALK depends on the EML4 region}

We then asked which fusion partner contributed to the phase separation of EML4-ALK. We created two truncation constructs and found that only GFP-EML4-N was able to 


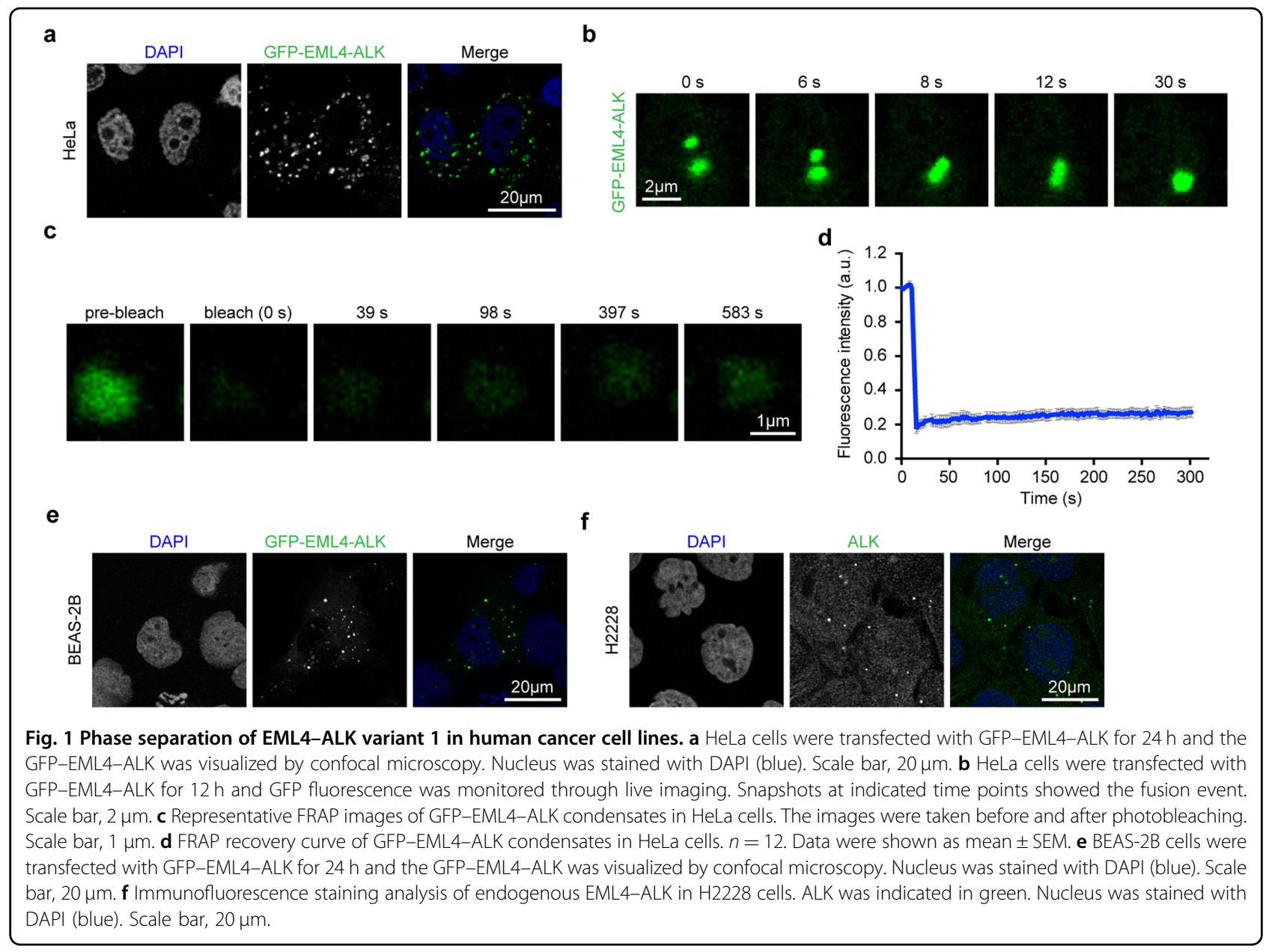

form condensates similar to GFP-EML4-ALK, whereas GFP-ALK-C showed a dispersed location in the cytoplasm (Fig. 4a). Through living cell imaging analyses, we found that the condensates of GFP-EML4-N also underwent fusion (Fig. 4b and Supplementary Movie S2). These findings indicate that EML4 region alone is sufficient for condensate formation.

EML4-ALK fusion leads to the constitutive activation of ALK kinase and results in the activation of downstream signaling pathways ${ }^{23}$. To further explore whether ALK kinase activity is necessary for condensate formation, we examined the dynamics of EML4-ALK condensates after ALK inhibitor treatment. Live imaging showed that either alectinib or ceritinib treatment had no significant impact upon the formation of EML4-ALK condensates (Fig. 4c, d). These data suggest that EML4-ALK condensate formation is potentially independent of its kinase activity.

Previous studies indicate that aromatic residues play an important role in promoting the phase separation of intrinsically disordered proteins ${ }^{24,25}$. We then generated the GFP-EML4-ALK21S mutant, in which most of the aromatic residues ( 9 tyrosine residues and 12 phenylalanine residues) were replaced in the EML4 region with serine residues (Supplementary Fig. S3). Western blot analysis showed comparable protein levels of GFP-EML4-ALK and GFP-EML4-ALK21S (Fig. 4e). In contrast to GFP-EML4-ALK, the GFP-EML4-ALK21S became dispersed in the cytoplasm when overexpressed in HeLa cells (Fig. 4f). These data support that the phase separation of EML4-ALK is dependent on the EML4 region.

\section{Phase separation is required for the EML4-ALK-induced hyperactivation of downstream signaling pathways}

As the phosphorylation of AKT, ERK1/2, and STAT3 are mainly mediated by three downstream signaling pathways of EML4-ALK ${ }^{16}$, we investigated whether the disruption of EML4-ALK condensate formation could affect the activation of EML4-ALK downstream signalings (Fig. 5a). Consistent with the previous report ${ }^{23}$, multiple cell lines stably expressing GFP-EML4-ALK displayed prominently elevated phosphorylation levels of AKT, ERK1/2, and STAT3 as compared to their parental control cells (Fig. 5b-d). In sharp contrast, the STAT3 phosphorylation levels were comparable between cells 
a

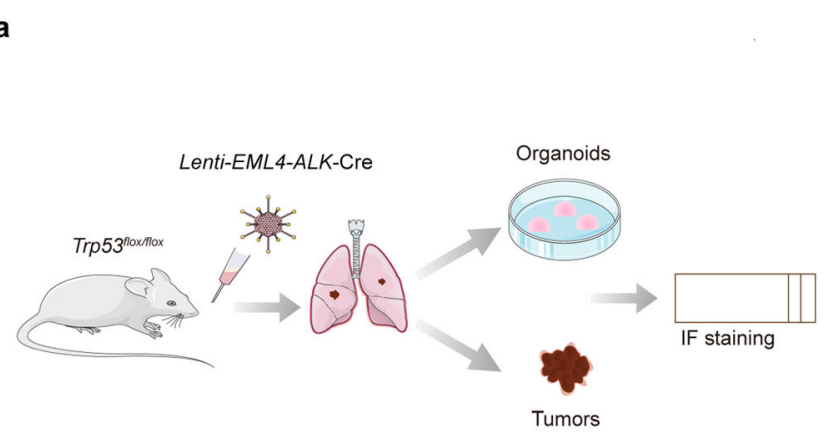

d
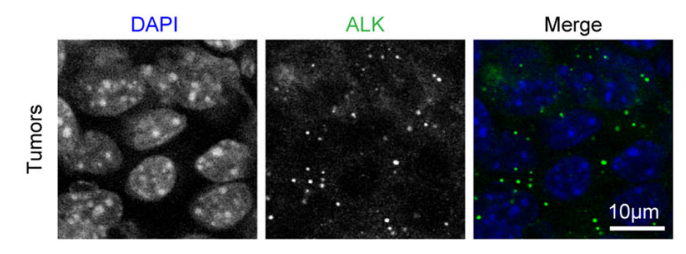

e b

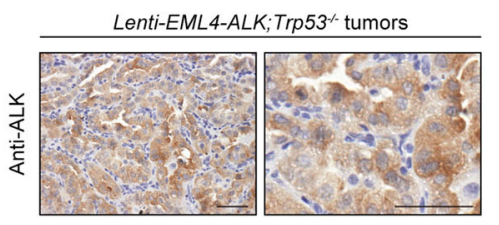

c

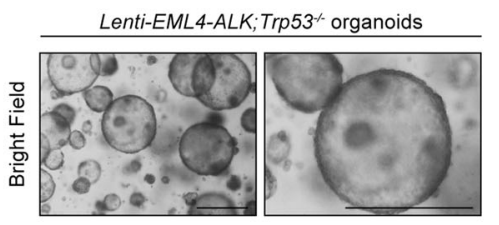

Fig. 2 EML4-ALK forms condensates in lung tumors and tumor-derived organoids in Lenti-EML4-ALK;Trp53 ${ }^{-1-}$ mouse model. a Schematic illustration of Lenti-EML4-ALK;Trp53 $3^{-1-}$ mouse model. Trp53 $3^{\text {floxflox }}$ mice at 6-8 weeks were treated with $1 \times 10^{6} \mathrm{PFU}$ of Lenti-EML4-ALK-Cre lentivirus via nasal inhalation and analyzed 28 weeks afterward for immunofluorescence staining of lung tumors and tumor-derived organoids. $\mathbf{b}$ Representative

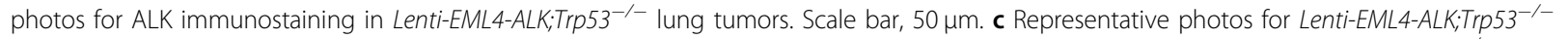
organoids derived from lung tumors. Scale bar, $500 \mu \mathrm{m}$. d Immunofluorescence staining analysis of EML4-ALK in Lenti-EML4-ALK; Trp53 ${ }^{-/-}$tumors. ALK was indicated in green. Nucleus was stained with DAPI (blue). Scale bar, $10 \mu \mathrm{m}$. e Immunofluorescence staining analysis of EML4-ALK in LentiEML4-ALK; Trp53 ${ }^{-/-}$organoids. ALK was indicated in green. Nucleus was stained with DAPI (blue). Scale bar, $10 \mu \mathrm{m}$.

stably expressing GFP-EML4-ALK21S and the control cells (Fig. 5b-d). The phosphorylation levels of ERK $1 / 2$ and AKT in the stable cell lines of constitutively expressing GFP-EML4-ALK21S were also comparable to or only slightly exceeded those of control cells (Fig. $5 \mathrm{~b}-\mathrm{d}$ ). These results indicate that the phase separation property of EML4-ALK is essential for its ability to hyperactivate these downstream pathways, especially STAT3 phosphorylation.

To further clarify the link between the STAT3 phosphorylation and the condensate formation, we overexpressed GFP-EML4-ALK and GFP-EML4-ALK21S in $\mathrm{H} 2228$ cells and performed immunostaining analysis. The overexpression of GFP-EML4-ALK led to the formation of condensates and the enrichment of p-STAT3 (Fig. 5e), whereas the overexpression of GFP-EML4-ALK21S failed to form condensates (Fig. 5f). This suggests a possibility that the EML4-ALK condensates hyperactivate the STAT3 signaling pathway through the direct recruitment of downstream component.

\section{Phase separation of EML4-ALK is critical for its oncogenic property}

We next functionally characterized whether the disruption of EML4-ALK condensate formation disturbed the neoplastic transformation. In contrast to wild-type
EML4-ALK, the EML4-ALK21S mutant displayed dramatically decreased capability in promoting soft-agar colony formation in NIH3T3 cells (Fig. 6a-c). Using Kras mouse embryonic fibroblasts (MEFs) and BEAS-2B cells, we observed similar decrease of transformation capabilities upon the overexpression of EML4-ALK21S mutant (Fig. 6d-i).

We further performed in vivo tumor formation assay in nude mice using NIH3T3 cells with overexpression of wild-type EML4-ALK or the EML4-ALK21S mutant (Fig. 7a). The wild-type EML4-ALK was able to drive fast tumor growth even after 11 days of transplantation (Fig. 7c). In contrast, the EML4-ALK21S mutant group showed a significantly impaired tumor growth and dramatically decreased tumor sizes and weights (Fig. 7b-d), despite of comparable ALK expression (Fig. 7e). The EML4-ALK condensates were clearly present in wild-type EML4-ALK tumors but almost undetectable in EML4-ALK21S tumors (Fig. 7f). Consistently, proliferative cells indicated by Ki-67 positive staining were significantly decreased in EML4-ALK21S tumors (Fig. 7g-i). To further check the changes of downstream signalings, we conducted the immunostaining of p-AKT, p-ERK1/2, and p-STAT3. Compared to wild-type EML4-ALK tumors, the EML4-ALK21S tumors showed markedly reduced pSTAT3 levels (Fig. 7j-l). The p-ERK1/2 levels were also 


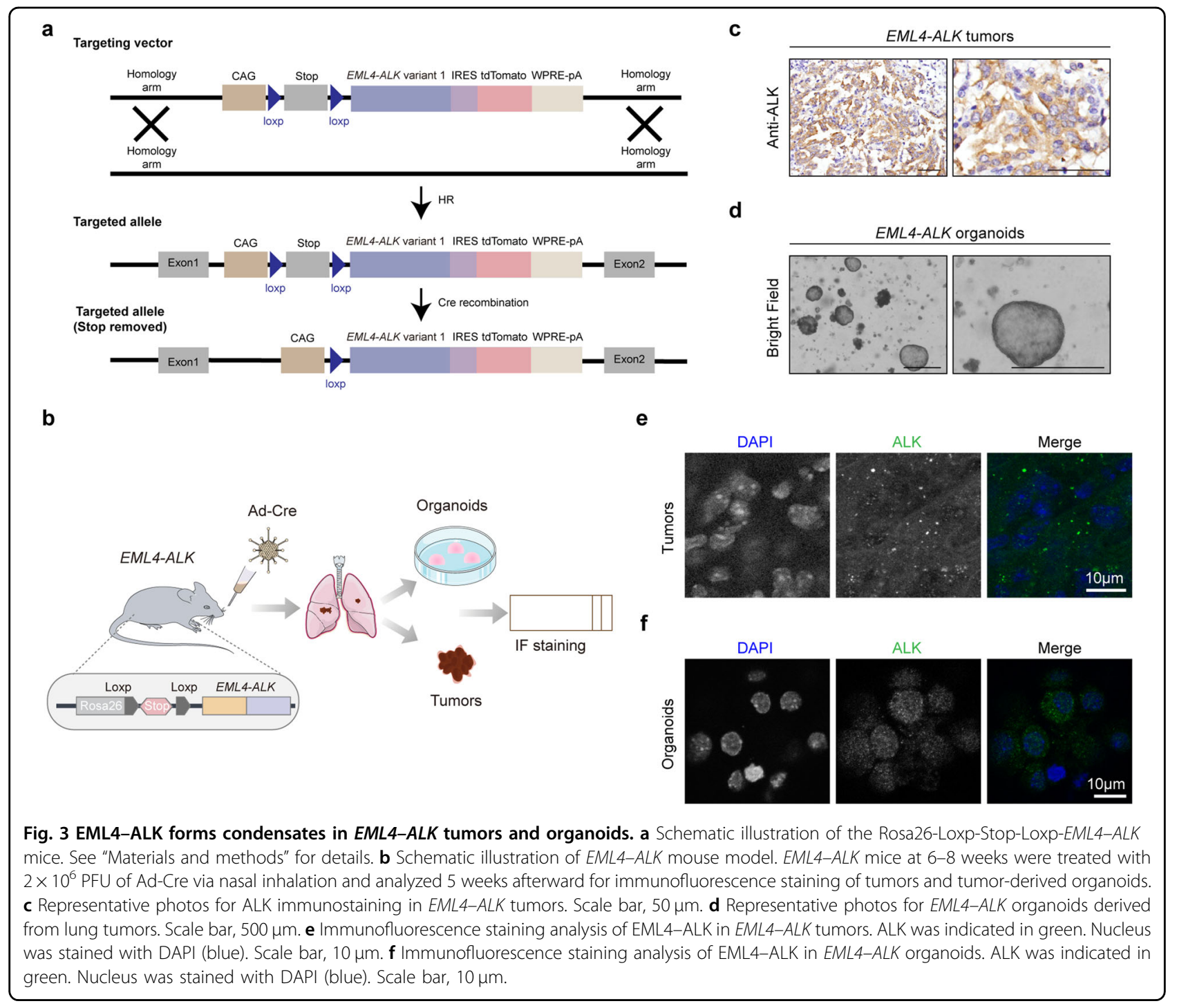

reduced but not as striking (Supplementary Fig. S4a-c), whereas the levels of p-AKT were barely detectable in all the tumors (Supplementary Fig. S4d). These data together demonstrate that EML4-ALK phase separation is required for the downstream STAT3 activation and neoplastic transformation.

\section{Discussion}

Emerging evidence begins to link cancer-related genes to condensate assembly, indicative of the important role of phase separation in tumorigenesis ${ }^{26}$. Boulay et al. find that the phase separation of EWS-FLI1 fusion promotes the formation of super-enhancers and oncogenic transcriptional programs in Ewing sarcoma cancer ${ }^{27}$. Another study links phase separation to tumor suppressor. Bouchard et al. find that mutations in the tumor suppressor $S P O P$ disrupt phase separation and lead to a buildup of cancer-promoting proteins $^{28}$. EML4-ALK fusion is the most dominant fusion in lung cancer. Previous studies report that kinase activities of EML4-ALK are mainly dependent on dimerization or autophosphorylation of the kinase domain ${ }^{29}$. Thus, it remains largely unknown whether phase separation contributes to the oncogenic activation of EML4-ALK.

Our study here shows that EML4-ALK forms condensates via phase separation in human cancer cell lines and mouse lung tumors. We further find that phase separation plays an important role in EML4-ALK-driven tumorigenesis whereas the disruption of phase separation significantly impairs downstream signaling and neoplastic transformation. Fascinatingly, two recent studies show that EML4-ALK variant 1 or variant 3 could form cytoplasmic protein granules to regulate downstream pathways $^{30,31}$. Tulpule et al. find low exchange of EML4-ALK variant 1 between the granules and the surroundings in FRAP assays ${ }^{31}$, which is consistent with our findings. Sampson et al. find that alectinib treatment promotes 


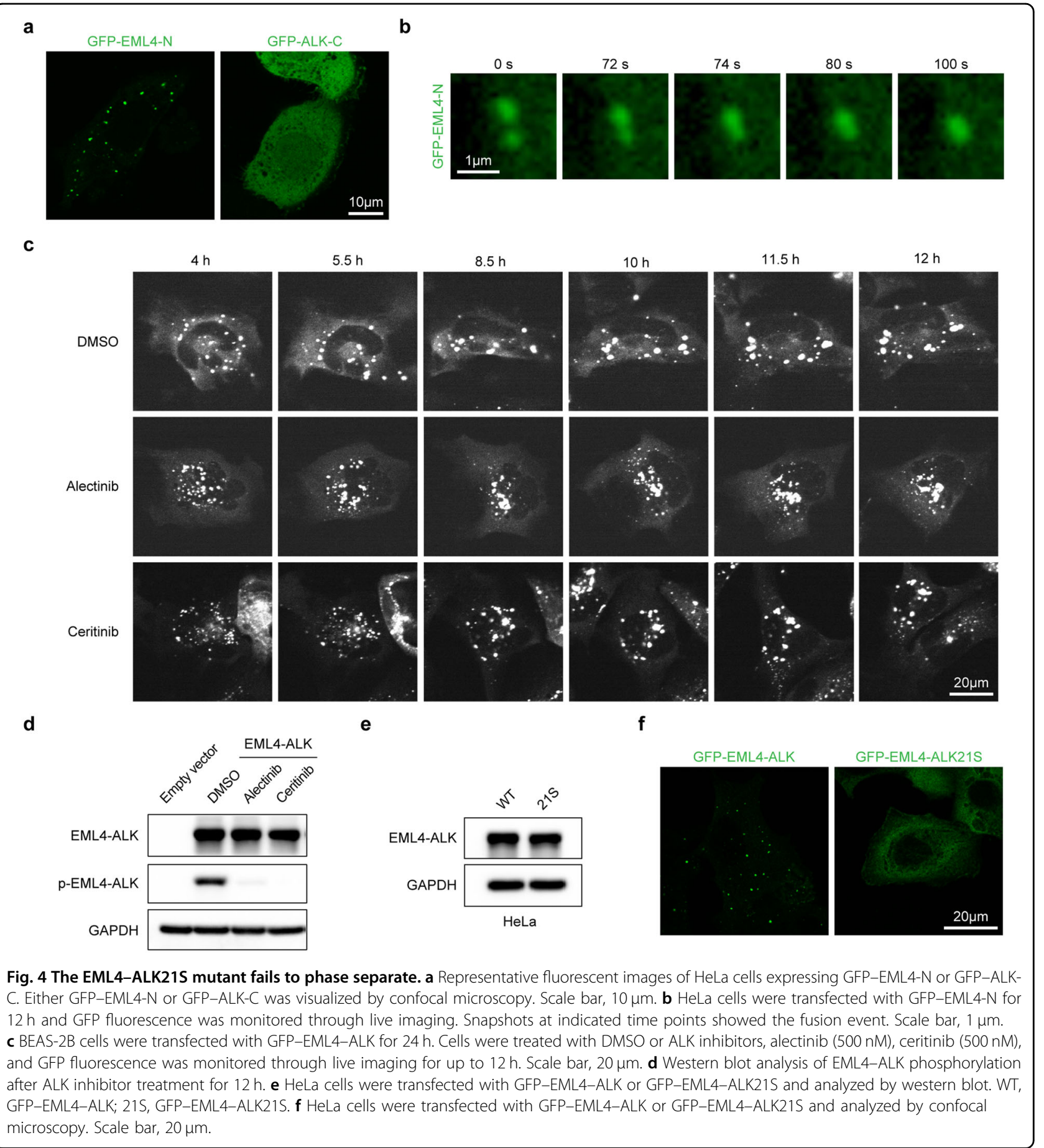

phase separation of EML4-ALK variant $3^{30}$. In contrast, we find that alectinib or ceritinib treatment shows no impact upon the phase separation of EML4-ALK variant 1 . We reason the effect of alectinib in phase separation might work in the context of different forms of EML4-ALK fusions. Future study will be interesting to elucidate the detailed relationship between EML4-ALK kinase activity and phase separation. Moreover, Tulpule et al. demonstrate that EML4-ALK granules mainly regulate downstream MAPK pathway ${ }^{31}$. Interestingly, we find although all the three pathways are affected by the disruption of phage separation, the decrease of STAT3 phosphorylation seems most dramatic in multiple cell lines and tumors. It remains very interesting to investigate how the phase separation finely tunes the various downstream signaling of EML4-ALK in the future. 


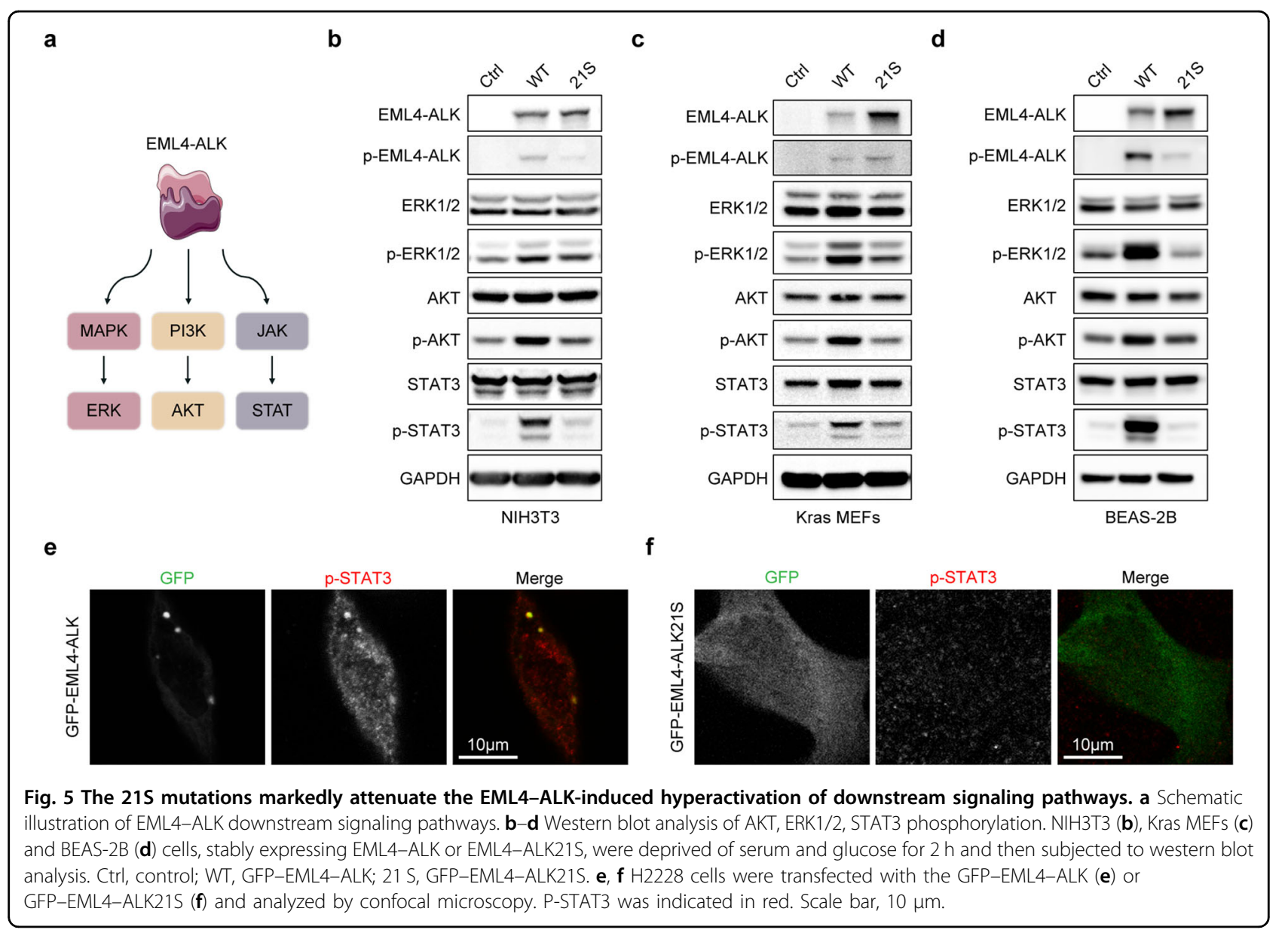

Collectively, our study demonstrates that phase separation is an important cellular process for EML4-ALK proteins and mediates the activation of downstream signaling pathways. Disruption of phase separation preferentially impairs the STAT3 phosphorylation and decreases the capability of malignant transformation. Our findings may provide a new approach for treating the EML4-ALK-positive lung cancer that aims to disrupt protein condensates.

\section{Materials and methods}

\section{Mouse model}

The $\operatorname{Tr} p 53^{f l o x}$ flox mice were originally provided by Dr. Tyler Jacks (Cambridge, MA). The transgenic EML4-ALK mouse model was generated by CRISPR/Cas9 technology. We inserted CAG-EML4-ALK-IRES-tdTomato expression box at the site of the Rosa26 gene through homologous recombination. The brief process is as follows: Cas9 mRNA and gRNA were obtained by in vitro transcription, the homologous recombination vector (donor vector) was constructed by the In-Fusion cloning method, which contained a $3.3 \mathrm{~kb} 5^{\prime}$ homology arm, CAG-EML4-ALKIRES-tdTomato, and $3.3 \mathrm{~kb} 3^{\prime}$ homology arm. Cas9 mRNA, gRNA, and donor vector were microinjected into the fertilized eggs of C57BL/6J mice to obtain F0 generation mice. All mice were kept in specific pathogen-free environment of Shanghai Institute of Biochemistry and Cell Biology, received humane care and treated in strict accordance with protocols approved by the Institutional Animal Care and Use Committee of the Shanghai Institutes for Biological Sciences, Chinese Academy of Sciences. Mice were treated with Ad-Cre virus at $2 \times 10^{6} \mathrm{PFU}$ or Lenti-EML4-ALK-Cre virus at $1 \times 10^{6}$ PFU by nasal inhalation at 6-8 weeks of age.

\section{Plasmid construction}

Full-length EML4-ALK was amplified and inserted into GFP-3x linker vector plasmid. Domain truncation constructs were generated by standard PCR-based cloning. Mutant plasmid was synthesized by Gene synthesis technology in shanghai Generay biotech Co., Ltd. All the constructs were verified by sequencing.

\section{Immunofluorescence and Fluorescent microscopy}

Cultured cells or organoids were fixed with $4 \%$ paraformaldehyde in PBS for $15 \mathrm{~min}$ at room temperature. Fixed cells were permeabilized with $0.5 \%$ Triton X-100 in 
a

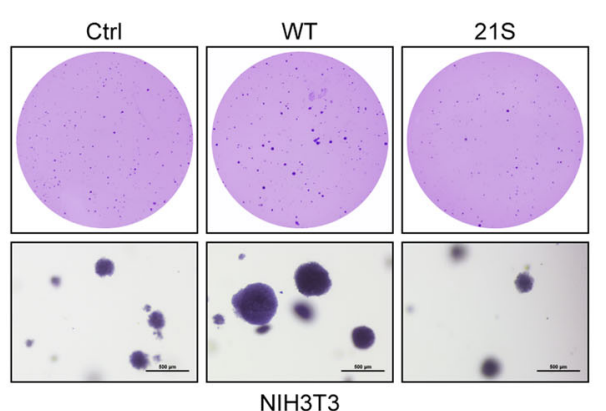

d

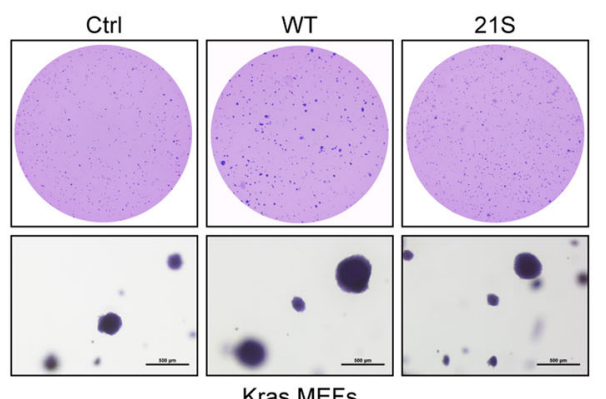

g

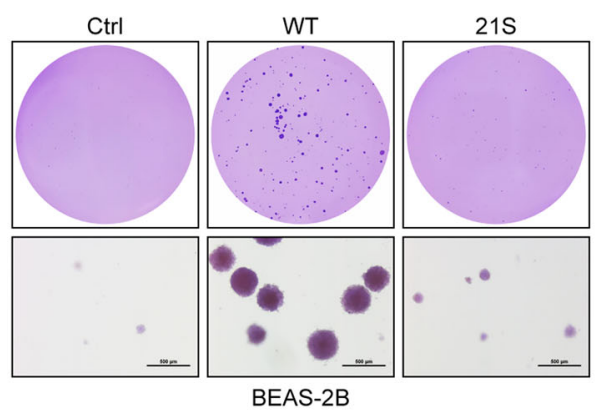

b

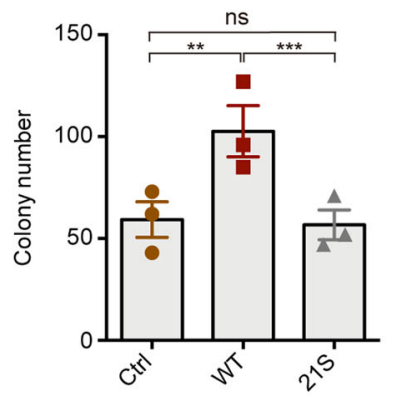

e

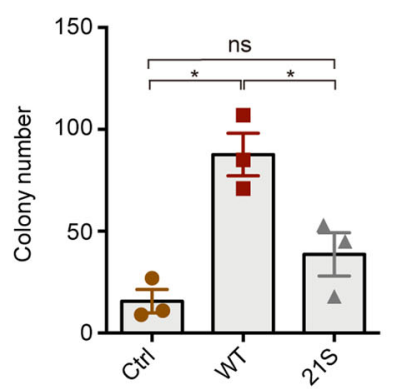

h

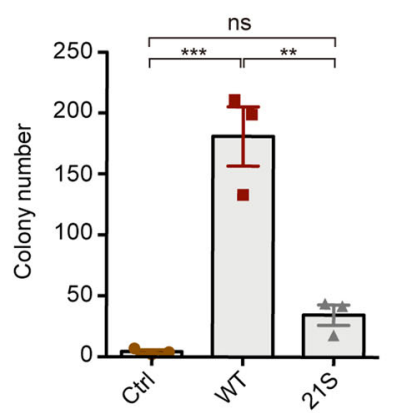

C

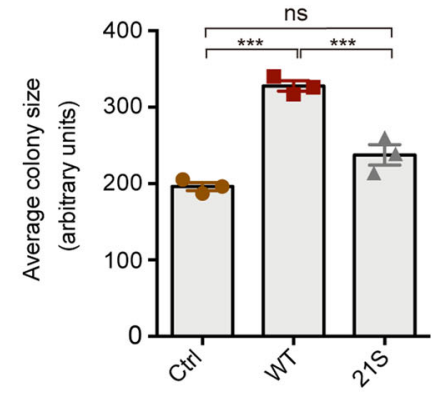

$f$

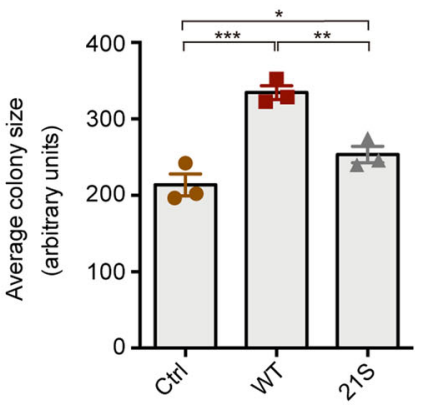

i

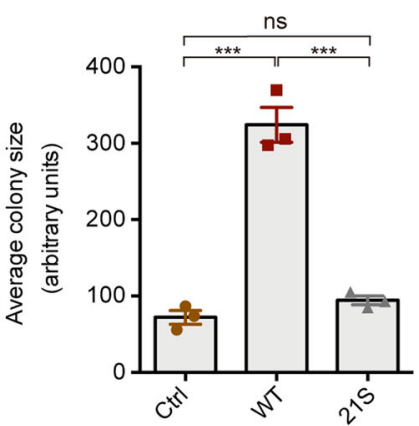

Fig. 6 The $21 \mathrm{~S}$ mutations impair the tumorigenic capability of EML4-ALK in soft-agar colony formation. a-c NIH3T3 cells were stably transfected with empty vector, EML4-ALK or EML4-ALK21S and analyzed by soft-agar colony formation assay. Representative images for soft-agar colonies (a). Scale bar, 500 um. Statistical analysis of colony numbers (b). Statistical analysis of colony sizes (c). d-f Kras MEFs cells were stably transfected with empty vector, EML4-ALK or EML4-ALK21S and analyzed by soft-agar colony formation assay. Representative images for soft-agar colonies (d). Scale bar, $500 \mu \mathrm{m}$. Statistical analysis of colony numbers (e). Statistical analysis of colony sizes (f). g-i BEAS-2B cells were stably transfected with empty vector, EML4-ALK or EML4-ALK21S and analyzed by soft-agar colony formation assay. Representative images for soft-agar colonies (g). Scale bar, $500 \mu \mathrm{m}$. Statistical analysis of colony numbers (h). Statistical analysis of colony sizes (i). All data were shown as mean \pm SEM. ${ }^{*} P<0.05$; ${ }^{* *} P<0.01$; ${ }^{* *} P<0.001$; ns: not significant. Ctrl, control; WT, GFP-EML4-ALK; 21S, GFP-EML4-ALK21S.

PBS for $15 \mathrm{~min}$ and blocked with $4 \%$ bovine serum albumin in TBST for $1 \mathrm{~h}$. The cells or organoids were incubated with following antibodies: ALK (CST, $3633 \mathrm{~S}$, 1:250), p-STAT3 (CST, 7145, 1:100) and washed three times with $4 \%$ bovine serum albumin. After incubation with secondary antibodies at room temperature for $1 \mathrm{~h}$, the cells were washed three times with TBST. Then, the coverslips were mounted onto glass slides using fluorescent mounting medium. Confocal images were captured using a Leica TCS SP8 system with a HC PL APO CS2 $63 \times / 1.40$ oil objective.

\section{Live-cell imaging}

For live-cell imaging, cells were seeded in $35-\mathrm{mm}$ glassbottom dishes (D35-20-1.5-N, Cellvis). For imaging the droplet fusion, HeLa cells were transfected with plasmids for $12 \mathrm{~h}$. Images were captured at $2 \mathrm{~s}$ intervals with a Zeiss LSM880 Airyscan microscope equipped with a $63 \times$ oil immersion objective. For live-cell imaging after ALK inhibitor treatment, BEAS-2B cells were transfected with plasmids for $24 \mathrm{~h}$ and treated with alectinib (MCE, $500 \mathrm{nM}$ ) and ceritinib (MCE, $500 \mathrm{nM})$. Continuous images were captured for $12 \mathrm{~h}$. 


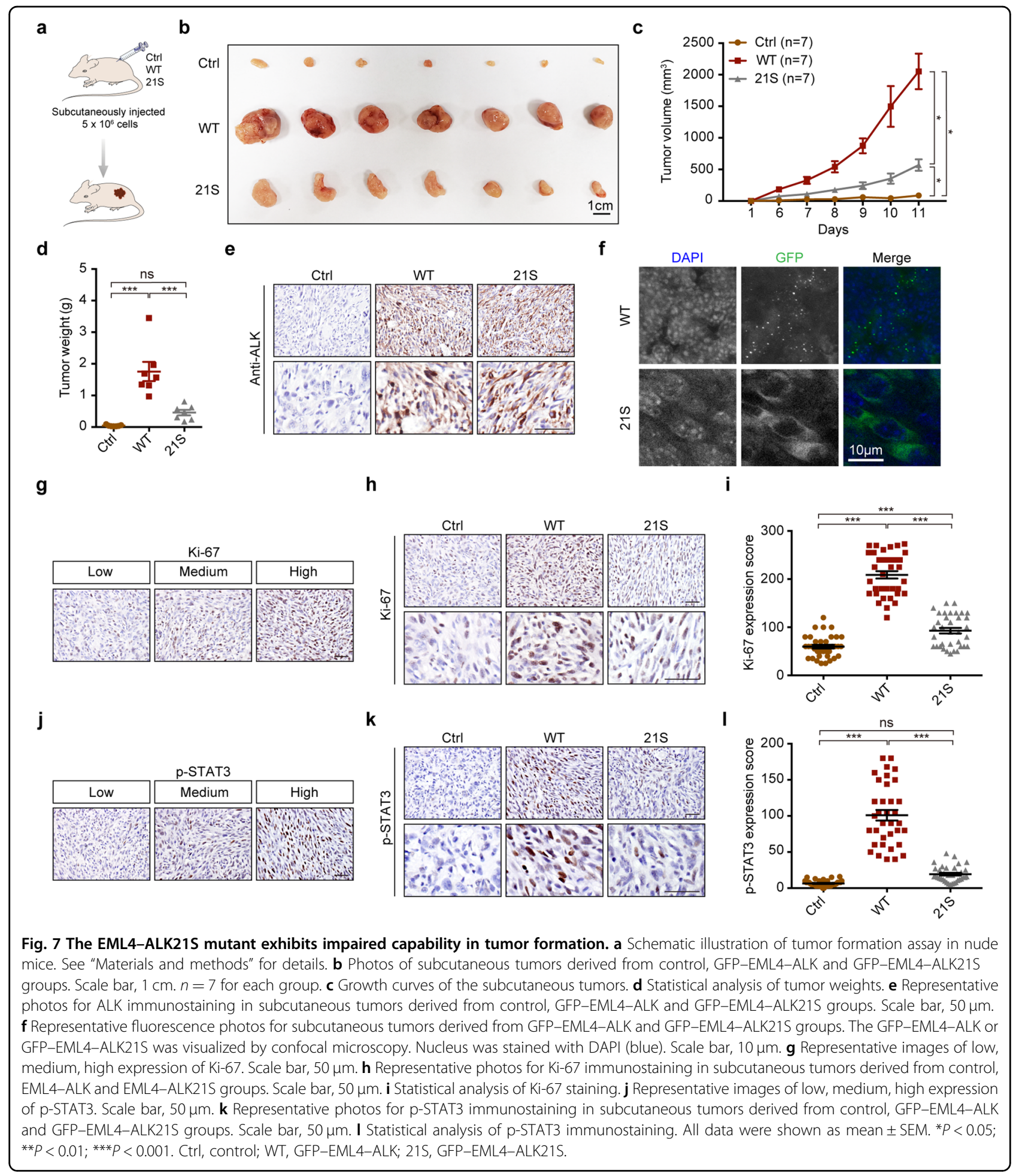

\section{Fluorescence recovery after photobleaching (FRAP)}

FRAP experiments in cells were carried out with following settings: region of interest (ROI) were bleached using a $405 \mathrm{~nm}$ diode, pre-bleach and postbleach images were acquired with a $488 \mathrm{~nm}$ laser.
Fluorescence recovery of GFP-EML4-ALK was monitored for 10 or $15 \mathrm{~min}$ with a time resolution of $2 \mathrm{~s}$. Images were captured at $2 \mathrm{~s}$ intervals with a Zeiss LSM880 Airyscan microscope equipped with a $63 \times$ oil immersion objective. 


\section{In vitro organoid culture}

The mouse tumor organoids were established using previously described culture methods ${ }^{32}$. Briefly, mouse tumors were minced with scissors and digested in $1 \mathrm{~mL}$ of $5 \mathrm{mg} / \mathrm{mL}$ collagenase type II (Invitrogen) in Advanced DMEM/F12 (Gibco) and digested for $1-2 \mathrm{~h}$ at $37^{\circ} \mathrm{C}$ with shaking. Dissociated cells were washed and then seeded in growth factor-reduced Matrigel (BD biosciences). Organoids were passaged at a 1:3 dilution every 4 days via trituration with glass Pasteur pipettes.

\section{Lentivirus production and infection}

The production of lentivirus supernatant was described previously $^{33}$. The cell lines NIH3T3 (ATCC) and Kras MEFs were maintained in DMEM (Hyclone) supplemented with $8 \%$ FBS, the cell lines BEAS-2B (ATCC) were maintained in RPMI-1640 supplemented with 8\% FBS. For stable overexpression of EML4-ALK variant 1, the cells infected with virus were persistently maintained in medium with puromycin $(2 \mu \mathrm{g} / \mathrm{mL}$, Sigma).

\section{Western blot}

Whole-cell lysates of cell lines were prepared in lysis buffer (10\% SDS, $1 \mathrm{mM}$ DTT, and glycerin) and incubated at $100^{\circ} \mathrm{C}$ for $10 \mathrm{~min}$. Equal volumes of proteins were resolved by SDS-PAGE and transferred onto PVDF membranes. Protein samples were probed with specific antibodies against ALK (CST, $3633 \mathrm{~S}, 1: 2000)$ ), p-ALK (CST, 3341S, 1:1000), ERK (CST, 9102, 1:1000), p-ERK (CST, 4370, 1:1000), AKT (CST, 9272, 1:1000), p-AKT (CST, 4070, 1:1000), STAT3 (CST, 9139, 1:1000), p-STAT3 (CST, 7145, 1:1000), or GAPDH (Abclonal, AC002, 1:5000). Protein expression was assessed by Pierce ECL Western Blotting Substrate (Thermo Fisher Scientific) and detected on SAGECREATION (Sage Creation Science Co, Beijing).

\section{Immunostaining}

Immunostaining was performed as previously descri$\operatorname{bed}^{34}$. Paraffin-embedded tissues were incubated with following antibodies: ALK (CST, 3633 S, 1:250), Ki-67 (Novus, NB500-170, 1:1000), p-ERK (CST, 4370, 1:400), p-AKT (CST, 4070, 1:400), p-STAT3 (CST, 7145, 1:400). The IHC expression score was evaluated by counting Ki-67-positive, p-STAT3-positive and p-ERK1/2-positive staining at highpower field (HPF) for 35 fields for each group.

\section{Soft agar colony formation assay}

For soft agar assay, a bottom layer of $1 \%$ agar with complete medium is solidified first, followed by an upper layer containing 5000 cells suspended in $0.4 \%$ mediumagar mixture in 6-well plates. After 2-3 weeks of incubation, cells were stained with $0.005 \%$ crystal violet and the number of colonies were counted. All experiments were performed in triplicates.

\section{Xenograft assay}

NIH3T3 cells with EML4-ALK or EML4-ALK21S expression were subcutaneously transplanted into nude mice $\left(5 \times 10^{6}\right.$ cells per mouse). Tumor volume was monitored every day and calculated by using formulation $\mathrm{V}=$ $(\mathrm{L} \times \mathrm{W} \times \mathrm{W}) / 2$. Mice were sacrificed and the tumors were harvested for further molecular and pathological analysis.

\section{Statistical analysis}

Differences between groups were analyzed by One-way ANOVA or Two-way ANOVA and performed by Prism GraphPad software. $P$ value $<0.05$ was considered statistically significant. Error bars were represented with SEM.

\section{Acknowledgements \\ This work was supported by the National Basic Research Program of China (2017YFA0505501, 2020YFA0803300 to H.J.), Strategic Priority Research Program of the Chinese Academy of Sciences (XDB19020201 to H.J. and XDB19020102 to X.Z.), National Natural Science Foundation of China (82030083, $31621003,81872312,82011540007$ to H.J., 81871875 to L.H., 81902326 to X.W., 81802279 to H.H.), Basic Frontier Scientific Research Program of Chinese Academy of Sciences (ZDBS-LY-SM006 to H.J.), International Cooperation Project of Chinese Academy of Sciences (153D31 KYSB20190035 to H.J.), the State Key Laboratory of Oncogenes and Related Genes Foundation (KF-20-03 to H.J.), the Youth Innovation Promotion Association CAS (Y919S31371 to X.W.).}

\begin{abstract}
Author details
${ }^{1}$ State Key Laboratory of Cell Biology, Shanghai Institute of Biochemistry and Cell Biology, Center for Excellence in Molecular Cell Science, Chinese Academy of Sciences, Shanghai, China. ${ }^{2}$ University of Chinese Academy of Sciences, Beijing, China. ${ }^{3}$ School of Life Science and Technology, Shanghai Tech University, Shanghai, China. ${ }^{4}$ Institute of Life and Health Engineering, Jinan University, Guangzhou, Guangdong, China. ${ }^{5}$ Life Sciences Institute, Zhejiang University, Hangzhou, Zhejiang, China. ${ }^{6}$ School of Life Science, Hangzhou Institute for Advanced Study, University of Chinese Academy of Sciences, Hangzhou, Zhejiang, China
\end{abstract}

\section{Author contributions}

H.J., X.Y., and X.Z. conceived the project. Z.Q. and H.S. designed the experiments, acquired the data, and performed the analysis. H.S. performed experiments related to phase separation. Z.Q. performed experiments including mice analyses, functional assays, and biochemical assays. M.Y., X.P., L.C., and X.F. provided technical supports. H.J., Z.Q., H.S., X.Y., and X.Z. wrote the manuscript.

\section{Conflict of interest}

The authors declare no competing interests.

\section{Publisher's note}

Springer Nature remains neutral with regard to jurisdictional claims in published maps and institutional affiliations.

Supplementary information The online version contains supplementary material available at https://doi.org/10.1038/s41421-021-00270-5.

Received: 30 January 2021 Accepted: 11 April 2021

Published online: 11 May 2021

\footnotetext{
References

1. Siegel, R. L., Miller, K. D. \& Jemal, A. Cancer statistics, 2020. CA. Cancer J. Clin. 70, 7-30 (2020).
} 
2. Chen, Y., Tang, W. Y., Tong, X. \& Ji, H. Pathological transition as the arising mechanism for drug resistance in lung cancer. Cancer Commun. 39, 1-13 (2019).

3. Ding, L. et al. Somatic mutations affect key pathways in lung adenocarcinoma. Nature 455, 1069-1075 (2008).

4. Soda, M. et al. Identification of the transforming EML4-ALK fusion gene in non-small-cell lung cancer. Nature 448, 561-566 (2007).

5. Horn, L. \& Pao, W. EML4-ALK: Honing in on a new target in non-small-cell lung cancer. J. Clin. Oncol. 27, 4232-4236 (2009).

6. Solomon, B., Varella-Garcia, M. \& Camidge, D. R. ALK gene rearrangements: a new therapeutic target in a molecularly defined subset of non-small cell lung cancer. J. Thorac. Oncol. 4, 1450-1454 (2009).

7. Della Corte, C. M. et al. Role and targeting of anaplastic lymphoma kinase in cancer. Mol. Cancer 17, 1-9 (2018).

8. Vernersson, E. et al. Characterization of the expression of the ALK receptor tyrosine kinase in mice. Gene Expr. Patterns 6, 448-461 (2006).

9. Eichenmüller, B., Everley, P., Palange, J., Lepley, D. \& Suprenant, K. A. The human EMAP-like protein-70 (ELP70) is a microtubule destabilizer that localizes to the mitotic apparatus. J. Biol. Chem. 277, 1301-1309 (2002).

10. Sabir, S. R., Yeoh, S., Jackson, G. \& Bayliss, R. EML4-ALK variants: Biological and molecular properties, and the implications for patients. Cancers (Basel) 9, 188 (2017).

11. Lin, J. J. et al. Impact of EML4-ALK variant on resistance mechanisms and clinical outcomes in ALK-positive lung cancer. J. Clin. Oncol. 36, 1199-1206 (2018).

12. Soda, M. et al. A mouse model for EML4-ALK-positive lung cancer. Proc. Natl Acad. Sci. USA 105, 19893-19897 (2008).

13. Pyo, K. H. et al. Establishment of a conditional transgenic mouse model recapitulating EML4-ALK-positive human non-small cell lung cancer. J. Thorac. Oncol. 12, 491-500 (2017).

14. Karachaliou, N. \& Rosell, R. Systemic treatment in EGFR-ALK NSCLC patients: second line therapy and beyond. Cancer Biol. Med. 11, 173-181 (2014).

15. Zhang, Q. et al. ALK phosphorylates SMAD4 on tyrosine to disable TGF- $\beta$ tumour suppressor functions. Nat. Cell Biol. 21, 179-189 (2019).

16. Mossé, Y. P., Wood, A. \& Maris, J. M. Inhibition of ALK signaling for cancer therapy. Clin. Cancer Res. 15, 5609-5614 (2009).

17. Alberti, S., Gladfelter, A.\& Mittag, T. Considerations and challenges in studying liquid-liquid phase separation and biomolecular condensates. Cell $\mathbf{1 7 6}$ 419-434 (2019).
18. Mitrea, D. M. \& Kriwacki, R. W. Phase separation in biology; Functional organization of a higher order Short linear motifs-the unexplored frontier of the eukaryotic proteome. Cell Commun. Signal. 14, 1-20 (2016).

19. Hyman, A. A., Weber, C. A. \& Jülicher, F. Liquid-liquid phase separation in biology. Annu. Rev. Cell Dev. Biol. 30, 39-58 (2014).

20. Boeynaems, S. et al. Protein phase separation: a new phase in cell biology. Trends Cell Biol. 28, 420-435 (2018).

21. Zhang, $H$. et al. Liquid-liquid phase separation in biology: mechanisms, physiological functions and human diseases. Sci. China Life Sci. 63, 953-985 (2020).

22. Li, F. et al. LKB1 Inactivation elicits a redox imbalance to modulate non-smal cell lung cancer plasticity and therapeutic response. Cancer Cell 27, 698-711 (2015).

23. Koivunen, J. P. et al. EML4-ALK fusion gene and efficacy of an ALK kinase inhibitor in lung cancer. Clin. Cancer Res. 14, 4275-4283 (2008).

24. Pak, C. W. et al. Sequence determinants of intracellular phase separation by complex coacervation of a disordered protein. Mol. Cell 63, 72-85 (2016).

25. Jiang, $\mathbf{H}$. et al. Phase transition of spindle-associated protein regulate spindle apparatus assembly. Cell 163, 108-122 (2015).

26. Jiang, S., Fagman, J. B., Chen, C., Alberti, S. \& Liu, B. Protein phase separation and its role in tumorigenesis. Elife 9, 1-27 (2020).

27. Boulay, G. et al. Cancer-specific retargeting of BAF complexes by a prion-like domain. Cell 171, 163-178.e19 (2017).

28. Bouchard, J. J. et al. Cancer mutations of the tumor suppressor SPOP disrupt the formation of active, phase-separated compartments. Mol. Cell 72, 19-36.e8 (2018).

29. Hallberg, B. \& Palmer, R. H. The role of the ALK receptor in cancer biology. Ann. Oncol. 27, iii4-iii15 (2016).

30. Sampson, J., Richards, M., Choi, J., Fry, A. \& Bayliss, R. Phase-separation of EML4 ALK variant 3 is dependent upon an active ALK conformation. Preprint at bioRxiv https://www.biorxiv.org/content/10.1101/2020.06.11.144485v1 (2020).

31. Tulpule, A. et al. Kinase-mediated RAS signaling via membraneless cytoplasmic protein granules. Cell 1-16 (2021) https://doi.org/10.1016/j.cell.2021.03.031.

32. Gao, D. et al. Organoid cultures derived from patients with advanced prostate cancer. Cell 159, 176-187 (2014).

33. Gao, Y. et al. LKB1 inhibits lung cancer progression through lysyl oxidase and extracellular matrix remodeling. Proc. Natl Acad. Sci. USA 107, 18892-18897 (2010).

34. Ji, H. et al. LKB1 modulates lung cancer differentiation and metastasis. Nature 448, 807-810 (2007). 